\title{
Prevalencia de Violencia de Género en mujeres que acuden a consultas de Atención Primaria
}

\author{
Luis Miguel Cano Martína, Alberto Berrocoso Martínez ${ }^{\mathrm{b}}$, Leonor de Arriba Muñoz \\ Rodrigo Bernaldo de Quirós Lorenzana ${ }^{\mathrm{b}}$, Juan Diego Alamar Provechoc ${ }^{\mathrm{c}}$, Ana Cardo Maza ${ }^{\mathrm{d}}$
}

\begin{abstract}
${ }^{a}$ Médico de Familia, Centro de Salud de Menasalbas (Toledo).

b Médico de Familia, Centro de Salud de Benquerencia (Toledo).

c Médico de Familia, Centro de Salud de Cofrentes (Valencia).
\end{abstract}

d MIR Ginecología y Obstetricia, Hospital General de Albacete.

Correspondencia: Luis Miguel Cano Martín, C/ Castellón n 1, 45161 Polán, Toledo, España. Telf.: 627938829, e-mail: luismcano@msn.com.

Recibido el 4 de diciembre de 2009.

Aceptado para su publicación el 11 de enero de 2010.

\section{RESUMEN}

Objetivos. Conocer qué proporción de las mujeres que consultan en atención primaria sufren situaciones de violencia doméstica, así como las características clínicas y sociodemográficas asociadas.

Diseño. Estudio observacional, descriptivo, transversal.

Emplazamiento. Consultas de atención primaria rurales y urbanas.

Participantes. Muestreo consecutivo de mujeres de 18 o más años, con pareja sentimental actual o que hubiesen tenido pareja en el pasado.

Mediciones y resultados principales. Se diseñó un cuestionario que incluía variables sociodemográficas, antecedentes patológicos y de consumo de tóxicos, respuesta a la versión abreviada del Woman Abuse Screening Tool (WAST), asi como un cuestionario autoadministrable sobre situaciones de abuso.

Un $26,2 \%$ (IC 95\%: 21,7-31,1\%) presentó un resultado positivo del WAST y $25,9 \%$ (IC 95\%: $21,3-30,9 \%)$ admitió situaciones de abuso, físico, emocional o sexual, actual (11,7\%) o pasado (17,4\%). La concordancia en las respuestas a ambos cuestionarios fue baja (Kappa: 0,53).

Mediante análisis de regresión logística se encontró que el modelo que mejor predice la existencia de abuso incluye las variables etnia (OR: 0,14 -IC 95\%: 0,06-0,34- para las "caucásicas españolas") y estado civil (OR: 0,10 -IC95\%: 0,04-0,24- y OR: 0,09 -IC95\%: 0,040,21 - para solteras/viudas y casadas/pareja estable, respectivamente, comparado con el grupo de separadas/divorciadas).

Conclusiones: Una elevada proporción de mujeres que consultan en atención primaria han sufrido situaciones de violencia en el ámbito doméstico. Además de la sospecha clínica, es necesario disponer de algún instrumento de cribado o detección precoz para el diagnóstico de violencia contra la mujer.

Palabras clave: Violencia contra la Mujer, Maltrato conyugal, Atención Primaria de salud.

\section{ABSTRACT}

Prevalence of gender violence in women who attend Primary Care Centres

Objectives. To determine what percentage of women who attend primary care clinics suffer domestic violence and the women's associated clinical and socio-demographic characteristics.

Design. Cross-sectional, observational, descriptive study.

Setting. Rural and urban primary care centres.

Participants. Consecutive sample of woman aged 18 years or over, who has a partner or had a partner in the past.

Meaurements and main results. A questionnaire was designed that included socio-demographic variables, disease history and history of substance abuse. The short version of the Woman Abuse Screening Tool (WAST), and a self-administered questionnaire on abuse situations were also used.

Twenty six point two percent (95\% Cl: $21.7-31.1 \%$ ) had a postive WAST result and $25.9 \%$ (95\% Cl: $21.3-30.9 \%$ ) admitted to physical, emotional and sexual abuse by their partner at the present time $(11.7 \%)$ or in the past $(17.4 \%)$ The concordance of the responses to the two questionnaires was low (Kappa: 0.53).

Logistic regression analysis revealed that the model that best predicts the existence of abuse included ethnic variables (OR: $0.14-95 \% \mathrm{Cl}$ : 0.06-0.34- for "Spanish Caucasian women") and marital status (OR: $0.10-95 \% \mathrm{Cl}$ : 0.04-0.24- and OR: $0.09-95 \% \mathrm{Cl}$ : 0.04-0.21- for single women/widows and married women/stable partner, respectively, compared with the separated/divorced group).

Conclusions: A high percentage of women who attend primary care centres have suffered domestic violence. In addition to clinical suspicion a screening or early detection tool is needed in order to diagnose domestic violence.

Key words: Violence against Women, Spouse Abuse, Primary Health Care. 


\section{INTRODUCCIÓN}

Desde que en 1996 la Organización Mundial de la Salud reconociera la violencia contra la mujer como un problema prioritario de salud pública y de primera magnitud ${ }^{1-8} \mathrm{e}$ instara a los estados miembros a evaluar su dimensión, no han dejado de realizarse esfuerzos en la mayoría de los países del mundo con el fin de poner de manifiesto su posible existencia, e intentar llegar a una identificación precoz del problema en los casos en que se produjera ${ }^{1}$.

En España, con la aprobación de la Ley Orgánica 1/2004 de 28 de Diciembre, de Medidas de Protección Integral contra la violencia de género ${ }^{8}$, se intenta actuar contra la violencia que se ejerce sobre las mujeres por parte de quienes sean o hayan sido sus cónyuges o de quienes estén o hayan estado ligados a ellas por relaciones similares de afectividad, aun sin convivencia, estableciéndose medidas de protección integral cuya finalidad es prevenir, sancionar y erradicar esta violencia, y prestar asistencia a sus víctimas.

En dicha ley se establecen medidas de sensibilización e intervención en el ámbito sanitario para optimizar la detección precoz y la atención física y psicológica de las víctimas, medidas encaminadas a la sensibilización y formación de los profesionales sanitarios, con el fin de mejorar e impulsar el diagnóstico precoz, la asistencia y la rehabilitación de la mujer en las situaciones de violencia de género a que se refiere esta ley, estableciéndose igualmente apartados de prevención e intervención integral de violencia de género en los distintos Planes Nacionales de Salud.

En España, la III Macroencuesta sobre la violencia contra las mujeres ${ }^{9}$, llevada a cabo por el Instituto de la Mujer en el año 2006, puso de manifiesto que, de las mujeres residentes en España de 18 o más años, un 3,6\% declaran haber sido víctimas de malos tratos durante el último año, y un $9,6 \%$ pueden ser consideradas técnicamente como maltratadas por sus parejas. En Castilla-La Mancha, estos porcentajes eran de un $3,4 \%$ y un $11,5 \%$ respectivamente. Asimismo, se refleja que la incidencia de maltrato declarado y considerado técnicamente es mayor en las mujeres extranjeras que en las españolas, así como entre las mujeres discapacitadas.

Diversos estudios sobre violencia contra las mujeres en el ámbito sanitario han puesto de manifiesto que las mujeres que sufren malos tratos hacen un mayor uso del sistema sanitario, y aproximadamente un $20 \%$ de las mujeres que consultan en los servicios sanitarios padece esta situación $n^{1-3,5,6}$, y aunque los servicios sanitarios deberían ser un punto clave para el abordaje y la detección precoz de este tipo de violencia, sólo se identifica un $1 \%$ del total esti- mado de casos de mujeres maltratadas ${ }^{3,5}$. La falta de formación, y el hecho de que aún puedan existir opiniones que no consideran la violencia contra la mujer como un problema de salud, podrían influir en esta baja detección ${ }^{3,5,6,10}$.

Ante la evidencia de estos datos, y dada nuestra privilegiada posición en el ámbito de la Atención Primaria ${ }^{3-6}$, se planteó este trabajo, con el objetivo de conocer la prevalencia de situaciones de violencia de género entre las usuarias de las consultas de Atención Primaria, así como las características clínicas y sociodemográficas asociadas.

\section{MATERIAL Y MÉTODO}

Se realizó un estudio observacional descriptivo transversal entre los meses de diciembre de 2008 y febrero de 2009 en consultas de atención primaria y puntos de atención continuada, rurales y urbanos, pertenecientes al Servicio de Salud de Castilla-La Mancha (Zona Básica de Salud de Menasalbas y Zona Básica de Salud de Santa María de Benquerencia, ambas situadas en el Área de Salud de Toledo) y al Servicio Valenciano de Salud (Zona Básica de Salud de Cofrentes, Valencia).

La población estudiada fueron mujeres de 18 o más años que acudieron por cualquier motivo a las consultas de atención primaria y al punto de atención continuada de referencia, con relación de pareja en ese momento o que la hubiesen tenido en algún momento de su vida (entendiendo por relación de pareja aquella relación de carácter sentimental mantenida por la mujer con un hombre). Se excluyó a las mujeres que no entendían el español, a las que no sabía leer y/o escribir castellano, a las que presentaban disfunciones cognitivas moderadas 0 graves ya diagnosticadas y a aquéllas que nunca hubiesen tenido pareja. Siguiendo las Normas Éticas y de Seguridad para la Investigación sobre Violencia Doméstica ${ }^{10}$, se excluyó también a aquellas mujeres que acudían acompañadas por su pareja.

Se realizó un muestreo consecutivo de mujeres con el fin de alcanzar un tamaño muestral estimado de 385 mujeres, calculado para una prevalencia estimada del $20 \%$, con una precisión del $5 \%$ y un nivel de confianza del $95 \%$, obteniéndose finalmente un tamaño muestral de 371 mujeres al terminar el plazo acordado para la recogida de datos.

Se recogieron variables de carácter sociodemográfico y variables relacionadas con la violencia contra la mujer. Para la recogida de datos se diseño un cuestionario (anexo 1) en el que se recogieron cuestiones generales de carácter sociodemográfico, preguntas referentes a antecedentes patológicos de la mujer, hábitos tóxicos, la versión corta 
del cuestionario WAST (Woman Abuse Screening Tool $^{4}$, cuestionario validado para la identificación precoz de posibles mujeres maltratadas entre las que acuden a los servicios de Atención Primaria), y el cuestionario autoadministrado creado por Ruiz Pérez et al en un estudio similar al planteado, en el que se recogía la existencia de situaciones relacionadas con la violencia de género y existencia de malos tratos, tanto en el momento actual como en situaciones del pasado.

Se propuso a las mujeres la realización de los cuestionarios una vez terminado el motivo de consulta por el cual habían acudido, explicándoles previamente el objeto de la encuesta, la confidencialidad y anonimato de las mismas, la posibilidad de no contestar aquellas cuestiones que no les parecieran adecuadas $u$ oportunas, y mostrando nuestra predisposición para la intervención si la mujer se veía reflejada o afectada en lo referente a alguna de las cuestiones. Una vez realizados los cuestionarios de forma autocumplimentada, en presencia de la mujer, y sin lectura previa, se guardaron en un sobre cerrado para su posterior análisis, con el fin de mantener la confidencialidad y anonimato.

Se realizó el análisis estadístico a través del programa SPSS 12.0, utilizando para su descripción los parámetros habituales (media, desviación típica, porcentajes) con un intervalo de confianza del $95 \%$. Igualmente, se realizó un análisis de regresión logística variable con el fin de intentar conocer la existencia de algún modelo que pudiese predecir la existencia de situaciones de abuso y violencia domestica.

\section{RESULTADOS}

De todas las mujeres a las que se invitó, solamente una rechazó participar en el estudio y dos se descartaron por datos incompletos de la encuesta, analizándose finalmente los datos referentes a 368 mujeres tras finalizar el plazo acordado para la recogida de datos.

\begin{tabular}{|c|c|c|}
\hline \multirow[t]{6}{*}{ Nacionalidad } & Española & $91,0 \%$ \\
\hline & Sudamericana & $4,3 \%$ \\
\hline & Europea Este & $2,7 \%$ \\
\hline & Europea Oeste & $0,5 \%$ \\
\hline & Magrebí & $1,1 \%$ \\
\hline & Otras & $0,3 \%$ \\
\hline \multirow[t]{4}{*}{ Etnia } & Caucásica & $92,1 \%$ \\
\hline & Gitana & $1,6 \%$ \\
\hline & Árabe & $1,1 \%$ \\
\hline & Otras & $5,2 \%$ \\
\hline \multirow[t]{2}{*}{ Medio } & Rural & $36,14 \%$ \\
\hline & Urbano & $63,86 \%$ \\
\hline \multirow[t]{4}{*}{ Estado civil } & Soltera & $25,3 \%$ \\
\hline & Casada/pareja de hecho & $62,8 \%$ \\
\hline & Separada/divorciada & $10,1 \%$ \\
\hline & Viuda & $1,9 \%$ \\
\hline \multirow{4}{*}{ Número de hijos } & Sin hijos & $29,9 \%$ \\
\hline & Un hijo & $21,7 \%$ \\
\hline & Dos hijos & $30,4 \%$ \\
\hline & Familia numerosa ( $\geq 3$ hijos) & $18,0 \%$ \\
\hline \multirow[t]{5}{*}{ Estudios } & Sin estudios & $1,6 \%$ \\
\hline & Lee y escribe & $3,5 \%$ \\
\hline & Estudios primarios & $40,2 \%$ \\
\hline & Estudios secundarios & $27,2 \%$ \\
\hline & Tituladas medias y superiores & $27,4 \%$ \\
\hline \multirow[t]{6}{*}{ Ocupación } & Directivos y propietarios & $7,4 \%$ \\
\hline & Cuadros intermedios & $12,6 \%$ \\
\hline & Trabajadores manuales cualificados y semicualificados & $10,4 \%$ \\
\hline & Trabajadores no cualificados & $33,7 \%$ \\
\hline & Amas de casa & $24,4 \%$ \\
\hline & Otros & $11,5 \%$ \\
\hline
\end{tabular}

Tabla 1. Variables sociodemográficas. 
Respecto a los datos de carácter sociodemográfico (tabla 1), la gran mayoría de las mujeres eran españolas, con una edad media de 40,6 años (DE: 12,9) y un rango de edad de 18 a 77 años, residiendo en su mayor parte en el medio urbano. Más de la mitad de las mujeres estaban casadas o con pareja de hecho, y un $29,9 \%$ no tenía hijos. Respecto al nivel de estudios, eran mayoría las mujeres con estudios primarios, y solamente un $1,6 \%$ de las encuestadas no tenía estudios. Las ocupaciones más frecuentes fueron las de "trabajo no cualificado" y "ama de casa".
Un 97,6\% de las mujeres respondió la versión corta del WAST (tabla 2). De ellas, un $40,9 \%$ refirió "mucha tensión" o "alguna tensión" en su relación de pareja, y un $34,5 \%$ de las encuestadas resolvía sus discusiones con "mucha dificultad" o "alguna dificultad". Con ello, y según el WAST, se identificó un $26,2 \%$ de mujeres con resultado positivo en el test de cribado de violencia contra la mujer en la pareja.

\begin{tabular}{cc}
\hline ¿Cómo describiría usted su relación con su pareja? \\
Mucha tensión & $7,8 \%$ \\
Alguna tensión & $33,1 \%$ \\
Sin tensión & $59,1 \%$ \\
\hline \multicolumn{2}{c}{ Resuelven sus discusiones con: } \\
Mucha dificultad & $7,5 \%$ \\
Alguna dificultad & $27 \%$ \\
Sin dificultad & $65,5 \%$ \\
\hline \multicolumn{2}{c}{ Puntuación WAST } \\
0 puntos & $50,7 \%$ \\
1 punto & $23,1 \%$ \\
2 puntos (positivo) & $26,2 \%$ \\
\hline
\end{tabular}

Tabla 2. Versión corta del WAST. Resultados.

De todas las encuestadas, un 98,4\% respondió alguna de las preguntas del cuestionario autoadministrable referente a la existencia de situaciones de violencia con su pareja, actual o pasada, observando que en un $23,3 \%$ de los casos la mujer había sufrido en alguna ocasión situaciones de maltrato. Un $4,21 \%$ de las mujeres reconoció haber sufrido abusos en el pasado y estar sufriendo situaciones de maltrato por su pareja actual (tabla 3).

\begin{tabular}{lccccc}
\hline & Algún tipo de abuso & Abuso físico & Abuso emocional & Abuso sexual & Más de un tipo de abuso \\
\hline Pareja actual & $11,6 \%$ & $3,9 \%$ & $11,1 \%$ & $1,9 \%$ & $3,53 \%$ \\
Pareja en el pasado & $17,3 \%$ & $8,0 \%$ & $17,1 \%$ & $6,2 \%$ & $8,42 \%$ \\
$\begin{array}{l}\text { Por pareja pasada y } \\
\text { por pareja actual }\end{array}$ & $4,21 \%$ & - & - & - & - \\
$\begin{array}{l}\text { En algún momento } \\
\text { de su vida } \\
\text { (actual o pasado) }\end{array}$ & $23,3 \%$ & - & & - & - \\
\hline
\end{tabular}

Tabla 3. Situaciones de abuso por alguna pareja, actual y/o pasada 
Respecto a su pareja actual, respondió un $97,8 \%$ de las encuestadas, objetivándose una prevalencia de algún tipo de violencia contra la mujer del $11,6 \%$, siendo el abuso emocional el más frecuente, seguido por el abuso físico y el abuso sexual.

Respecto a otras parejas en el pasado, respondió un $92,4 \%$ de las encuestadas, contestando afirmativamente a alguno de los ítems de violencia un $17,3 \%$ de las mujeres y siendo más frecuente el abuso emocional. Un 5,2\% de las mujeres refirió no tener apoyo social.

Valorando los resultados del WAST como método de detección precoz de malos tratos, se obtuvo una sensibilidad del $100 \%$ (todas las mujeres que sufrían maltrato actual obtuvieron un resultado positivo en el WAST) y una especificidad del $84 \%$. Para una prevalencia de maltrato declarado del $11,6 \%$ en nuestra muestra, el valor predictivo positivo fue del $21,4 \%$, y el valor predictivo negativo fue del $100 \%$, existiendo una baja concordancia entre los dos test, WAST y Ruiz Pérez et al (índice kappa: 0,53)

Mediante análisis de regresión logística, se objetivó que el modelo que mejor predice una respuesta positiva con relación a la existencia de abuso (ya sea actual o pasado), incluye las variables "etnia", con menor riesgo en "caucásicas españolas" frente al resto de etnias y nacionalidades, y la variable "estado civil", con mayor riesgo en separadas/divorciadas respecto a solteras, viudas y aquéllas que tienen pareja estable (tabla 4). En nuestra muestra no había diferencias por nivel de estudios, ni por ocupación de la mujer o el cónyuge, como tampoco se pudo considerar factor de riesgo para maltrato actual el haber sufrido maltrato en el pasado.

\begin{tabular}{|c|c|c|}
\hline & OR & IC 95\% \\
\hline Etnia "caucásica española" & 0,142 & $0,059-0,342$ \\
\hline Estado civil "soltera -viuda" vs "separada-divorciada" & 0,099 & $0,40-0,246$ \\
\hline Estado civil "pareja estable" vs "separada-divorciada" & 0,09 & $0,039-0,21$ \\
\hline
\end{tabular}

Tabla 4. Modelo que mejor predice situaciones de violencia de género (actuales o pasadas).

\section{DISCUSIÓN}

Antes de comenzar a discutir los resultados de este trabajo, debemos señalar algunas posibles limitaciones del estudio. Así, alguno de los criterios de exclusión utilizados para seleccionar nuestra muestra puede llevarnos a infraestimar la prevalencia de maltrato en las mujeres, como ocurre con la exclusión de mujeres que acuden acompañadas por sus parejas, siguiendo las Normas Éticas y de Seguridad para la Investigación sobre la Violencia Doméstica ${ }^{10}$.

Igualmente ocurre con la exclusión de mujeres que no saben leer y/o escribir castellano y las que no entienden español, a pesar de que está demostrada una mayor prevalencia de maltrato en los niveles socioculturales más bajos y una mayor incidencia de mujeres que se declaran maltratadas y que pueden ser consideradas técnicamente como maltratadas entre las mujeres extranjeras residentes en España ${ }^{9,11}$, muchas de las cuales cumplen ese criterio de exclusión. Fueron excluidas por este concepto 18 mujeres, la mayor parte de origen magrebí, lo que puede disminuir la prevalencia de violencia de género de nuestro estudio respecto a este colectivo en particular, y la muestra en general.

Del mismo modo, los sesgos habituales de los estudios autorreferidos pueden hacernos infraestimar la prevalencia real de maltrato en nuestra población. También es preciso tener en cuenta, a la hora de valorar los datos, el hecho de que la muestra de este estudio podría no ser del todo representativa de la población general en lo referente a la proporción de mujeres representadas respecto a las diferentes variables estudiadas (nacionalidad, etnia, ocupación, estado civil, etc.).

Conocer la prevalencia real de violencia doméstica es tarea sumamente complicada, existiendo diferentes estudios al respecto. Algunos trabajos ${ }^{1-3}$ indican cifras de maltrato de entre el $10 \%$ y el $69 \%$, mientras que el estudio multipaís de la $\mathrm{OMS}^{12}$ sobre salud de la mujer y violencia doméstica contra la mujer eleva estas cifras, e indica que el porcentaje de mujeres que han tenido pareja alguna vez y que han sufrido violencia física o sexual, o ambas, por 
parte de su pareja a lo largo de su vida oscila entre el $15 \%$ y el $71 \%$ según países y zonas estudiadas, y que este fenómeno aparece sin excepción en todos los países del estudio.

En España, según la III Macroencuesta sobre la violencia contra las mujeres ${ }^{9}$, llevada a cabo por el Instituto de la Mujer en el año 2006, existen importantes diferencias entre las cifras de maltrato declarado y maltrato técnicamente considerado, con cifras más elevadas en el segundo caso. En nuestro trabajo hemos observado una prevalencia de un $11,6 \%$, que podemos considerar "maltrato declarado". Ante esta cifra surgen dos cuestiones. La primera sería: ¿por qué hemos detectado más en nuestro trabajo que en la macroencuesta? Quizá el hecho de preguntar a la mujer sobre el problema mediante un cuestionario, y no de forma verbal y "directa", ayude a la hora de reconocer la existencia del mismo, ya que las mujeres prefieren las preguntas escritas al interrogatorio directo ${ }^{13-15}$ a la hora de abordar este problema por parte del profesional sanitario.

La segunda cuestión sería la siguiente: si la prevalencia de maltrato actual o maltrato reconocido es la encontrada ¿cuál será la cifra de las que podemos considerar técnicamente maltratadas? $O$ visto desde otro punto de vista: ¿cuántas mujeres no declaran esta situación? Y es que, aunque hasta un $20 \%$ de las mujeres que utilizan los servicios sanitarios padece esta situación ${ }^{3,5,6}$, la detección por parte de los profesionales sanitarios no es la deseable, y ni siquiera el hecho de que la mayoría de la población del estudio pertenece a un medio urbano, donde aumenta el número de mujeres que se declara como maltratadas y hay un aumento significativo en la incidencia de violencia respecto a los municipios más pequeños ${ }^{9}$, hace que la cifra de detección de maltrato actual de nuestra muestra se acerque a ese umbral del $20 \%$ antes comentado. Atribuir este hecho a las limitaciones y posibles sesgos antes referidos, y no hacer autocrítica acerca de las razones de esta infradetección, sería poco profesional y poco ético por nuestra parte.

Se conocen incluso las "candidatas" a sufrir situaciones de violencia doméstica o violencia de género. Varios estudios ${ }^{11}$ ponen de manifiesto que la violencia del compañero íntimo se manifiesta de manera desigual y con mayor riesgo en los colectivos de mujeres con menor nivel educativo, mayor número de menores a su cargo y extranjeras, hablando incluso de la noción de "poblaciones vulnerables"11, subgrupos de población que, por compartir características sociales, tienen unas altas probabilidades de acumular más factores de riesgo que otros gru- pos de población, como ocurriría en estas mujeres respecto a la violencia de género. Esto se refleja en los resultados de nuestro trabajo, donde se objetiva una mayor probabilidad para sufrir situaciones de abuso entre las mujeres extranjeras. Igualmente hemos observado una mayor probabilidad entre las separadas y divorciadas, hecho que podríamos considerar como "esperable", ya que el haber sufrido maltrato en el pasado es considerado un factor de riesgo para una nueva situación de maltrato ${ }^{11}$. Sin embargo, no podemos considerar ambos aspectos como factores de riesgo en nuestra muestra, a pesar de lo reflejado en la bibliografía ${ }^{11}$. Quizá habría que aumentar el tamaño muestral para demostrar este hecho en nuestro estudio.

Y ante toda esta lluvia de datos nos surge la pregunta: si sabemos que el problema de salud es más frecuente de lo que dicen las encuestas, que permanece oculto, que las mujeres no se lo dicen directamente al profesional sanitario y que los profesionales tenemos múltiples barreras para afrontar el problema ${ }^{7}$, incluso conociendo aquéllas con más probabilidades para sufrir situaciones de violencia de género ${ }^{9,11}$, y aún así no lo detectamos, ¿por qué no hacer un cribado sistemático de su existencia en todas las mujeres que acuden a consulta, como hacemos con muchos otros problemas de salud?

Muchas son las controversias que han rodeado y rodean la idea de realizar un cribado sistemático de la posible existencia de malos tratos desde Atención Primaria, y una señal de estas controversias son los múltiples sistemas existentes para este cribado $^{13-16}$. Las diversas sociedades no han llegado a acuerdos concretos a este respecto, y así en los Estados Unidos existen opiniones discrepantes ${ }^{17}$. Mientras la American Medical Association (AMA) o el American College of Obstreticians and Gynecologist recomiendan que toda paciente mujer adulta debe ser interrogada de forma rutinaria acerca de la posible existencia de abuso o maltrato ${ }^{17}$, las guías basadas en la evidencia de entidades como la US Preventive Services Task Force o la Canadian Task Force on Preventive Health Care, concluyen que hay "evidencias insuficientes" de los beneficios para la salud de las supervivientes a abusos como para hacer recomendaciones a favor o en contra del cribado sistemático de lo que ellos llaman "violencia contra el compañero íntimo" ${ }^{17,18}$.

En España, el programa PAPPS de la Sociedad Española de Medicina Familiar y Comunitaria (SEMFYC) no encuentra evidencias suficientes para recomendar el cribado sistemático en la población general mediante cuestionarios específicos, aunque sí recomienda incluir preguntas sobre la 
existencia de maltrato en el curso de la entrevista clínica que pueda ayudar a su detección ${ }^{19,20}$. Mientras, otras sociedades como la Sociedad Española de Médicos de Atención Primaria (SEMERGEN) sí apuestan por el cribado sistemático ${ }^{21}$.

A nivel institucional, el Ministerio de Sanidad y Política Social recomienda, respecto a la detección precoz, que "el personal sanitario debe tener una actitud de alerta ante la presencia de conductas, síntomas o signos de sospecha". Además, se recomienda que en la primera visita de cada mujer, al iniciar la historia clínica, se realicen preguntas exploratorias de abordaje psicosocial ${ }^{22}$.

$Y$ si nos referimos a las diferentes Comunidades Autónomas, en algunas como Cantabria el protocolo elaborado propone la detección sistemática, a través de alguna pregunta específica en relación al tema, a todas las mujeres mayores de 14 años al abrir su historia de salud ${ }^{23,24}$, en un protocolo ya implantado en todos los Equipos de Atención Primaria e incluido en la Cartera de Servicios de Atención Primaria. Otras comunidades autónomas, como Madrid o Cataluña, han desarrollado instrumentos de cribado proactivo y diagnóstico para los distintos tipos de violencia a mujeres entre 18 y 70 años $^{23}$.

Ante la ausencia de consenso, si repasamos la bibliografía para encontrar una respuesta a nuestra pregunta, encontramos múltiples estudios que demuestran el beneficio del cribado sistemático de malos tratos desde Atención Primaria, pero estos resultados no son concluyentes a la hora de recomendar un cribado universal. Entonces, ¿a qué nos atenemos?

A la luz de nuestros resultados, aun teniendo en cuenta las limitaciones y posibles sesgos de nuestro trabajo, debemos concluir que, desde nuestro punto de vista, es recomendable la utilización de algún método de cribado de violencia contra la mujer en nuestras consultas, además de la sospecha clínica que siempre debe estar presente, con el fin de realizar un diagnostico lo más precoz posible de una situación de maltrato contra la mujer. En este sentido, destaca el cuestionario WAST abreviado como método de cribado de situaciones de violencia contra la mujer, cuestionario validado para tal $\mathrm{fin}^{4}$.

\section{BIBLIOGRAFÍA}

1. Ruiz-Pérez I, Plazaola-Castaño J, Blanco-Prieto P, González Barranco JM, Ayuso Martín P, Montero-Piñar MI y el Grupo de Estudio para la Violencia de Género. La violencia contra la mujer en la pareja. Un estudio en el ámbito de la atención primaria. Gac Sanit. 2006; 20(3):202-8.

2. Blanco P, Ruiz-Jarabo C, García de Vinuesa L, Martín-García M. La violencia de pareja y la salud de las mujeres. Gac Sanit. 2004; 18(Supl 1):182-8.
3. García Torrecillas JM, Torío Durántez J, lea Pereira MC, García Tirado MC, Aguilera Tejero R. Detección de violencia contra la mujer en la consulta del médico de familia. Aten Primaria. 2008; 40(9):455-61.

4. Piazola-Castaño J, Ruiz Pérez I, Hernández-Torres E. Validación de la versión corta del Woman Abuse Screening Tool para su uso en atención primaria en España. Gac Sanit. 2008; 22(5):451-60.

5. Coll-Vinent Puig B, Echeverría T, Farrás U, Rodríguez D, Millás J, Santiñá M. El personal sanitario no percibe la violencia doméstica como un problema de salud. Gac Sanit. 2008; 22(1):7-10.

6. Coll-Vinent Puig B. Identificación en urgencias de los casos de violencia doméstica. Jano. 2006; 1596:39-41.

7. Siendones Castillo R, Perea-Milla López E, Arjona Huertas JL, Agüera Urbano C, Rubio Gallo A, Molina Molina M. Violencia doméstica y profesionales sanitarios: conocimientos, opiniones y barreras para la infradetección. Emergencias. 2002; 14:224-32.

8. Ley Orgánica $1 / 2004$, de 28 de diciembre, de Medidas de Protección Integral contra la Violencia de Género. BOE núm. 313, Miércoles 29 diciembre 2004. Pag. 42166-97.

9. Instituto de la Mujer. Ministerio de Trabajo y Asuntos Sociales. La violencia contra las mujeres. Resultados de la III Macroencuesta. Madrid: Sigma Dos S.A; 2006. Disponible en: http://mtas.es.

10. World Health Organisation. Putting women's safety first; ethical and safety recommendations for research on domestic violence against women. Global Programme on Evidence for Health Policy Gen? World Health Organisation; 1999.

11. Vives-Cases C, Álvarez-Dardet C, Gil-González D, Torrubiano-Domínguez J, Rohlfs I, Escribà-Agüir V. Perfil sociodemográfico de las mujeres afectadas por violencia del compañero íntimo en España. GacSanit. 2009; 23(5):410-4.

12. Estudio multipaís de la OMS sobre salud de la mujer y violencia doméstica contra la mujer. Organización Mundial de la Salud; 2005. Disponible en: http://who.it/gender.

13. McCord-Duncan E, Floyd M, Kemp E, Bailey b, Lang F. Detecting potential intimate partner violence: which approach do women want? Fam Med. 2006; 38(6):416-22.

14. Chen P, Rovi S, Washington J, Jacobs A, Vega M, Pan K et al. Randomized comparison of 3 methods to screen for domestic violence in family practice. Ann Intern Med 2007; 5:430-5.

15. MacMillan H, Wathen C, Jamieson E, Boyle M, McNutt L, Worster A et al. Approaches to screening for intimate partner violence in health care settings: a randomized trial. JAMA. 2006; 296(5):530-6.

16. Catálogo de instrumentos para cribado y frecuencia del maltrato físico, psicológico y sexual. Unidad de apoyo a la Investigación. Escuela Andaluza de Salud Pública, para Observatorio de Salud de la Mujer; 2007. Disponible en: http:// www.msps.es/organizacion/sns/planCalidadSNS/pdf/equidad/genero_vg_01.pdf.

17. Moracco K, Cole T. Preventing intimate partner violence: screening is not enough. JAMA. 2009; 302(5):568-70.

18. MacMillan $H$, Wathen $C$, Jamieson $E$, Boyle $M$, Shannon $H$, Ford-Gilboe $\mathrm{M}$ et al. Screening for intimate partner violence in health care settings: a randomized trial. JAMA. 2009; 302(5):493-501.

19. Grupo de Salud Mental del Programa de Actividades de Prevención y Promoción de la Salud (PAPPS) de la Sociedad Española de Medicina de Familia y Comunitaria (SEMFYC), con la colaboración del Ministerio de Sanidad y Consumo. Violencia Doméstica. Madrid: Ministerio de Sanidad y Consumo; 2003. Disponible en: http://www.msps.es/ciudadanos/violencia/docs/violenciadomestica.pdf.

20. Fernández Alonso MC, Buitrago Ramírez F, Ciurana Misol R, Chocrón Bentata L, García Campayo J, Montón Franco 
C et al. Grupo de salud mental del PAPPS. Programa de prevención en salud mental en atención primaria. Aten Primaria. 2007; 39(Supl 3):88-108.

21. Martín Prieto M, González Rodríguez MA, Vicente Muelas N. El Maltrato. Madrid: SEMERGEN; 2005. Disponible en: http://www.semergen.es/semergen/microsites/manuales/ maltrato/maltrato.htm.

22. Protocolo común para la actuación sanitaria ante la violencia de género. Comisión Contra la Violencia de Género del Consejo Interterritorial del Sistema Nacional de Salud. Ministerio de Sanidad y Consumo; 2007. Disponible en: http://
www.msc.es/organizacion/sns/planCalidadSNS/pdf/equi$\mathrm{dad} /$ protocoloComun.pdf.

23. Informe violencia de género 2006. Informes, estudios e investigación 2008. Ministerio de Sanidad y Consumo. Disponible en: http://www.msc.es/organizacion/sns/planCalidadSNS/pdf/equidad/informeViolenciaGenero2006.pdf.

24. Violencia contra las mujeres. Protocolo de actuación sanitaria ante los malos tratos. Gobierno de Cantabria. Consejería de Sanidad y Servicios Sociales. Dirección General de Salud Pública; 2005. Disponible en: http://www.saludcantabria. org/saludPublica/pdf/mujeres/ProtocoloViolencia.pdf.

\section{ANEXO 1. CUESTIONARIO AUTOADMINISTRADO}

Este cuestionario forma parte de un estudio sobre salud y mujer

Las respuestas al mismo son totalmente confidenciales, garantizándose el anonimato de las mismas.

$\mathrm{N}^{\circ}$ identificación:

Edad:

Número de hijos:

española sudamericana europea este

europea oeste magrebí subsahariana

Etnia: caucásica gitana

Estado civil: soltera

casada/pareja de hecho separada/divorciada

negra

otras

Estudios: $\quad$ sin estudios lee y escribe

primarios secundarios

viuda

Ocupación:

Ocupación cónyuge (o cabeza de familia en no emancipadas):

Situación laboral: activa parada

no realiza trabajo remunerado

jubilada

Antecedentes patológicos:

Fumadora: SI

SI NO

Exfumadora

Alcohol:

NO

SI

NO

Versión corta del Woman Abuse Screening Tool (WAST)

-En general, ¿cómo describiría usted su relación con su pareja?

Mucha tensión Alguna tensión

Sin tensión

-Usted y su pareja resuelven sus discusiones con:

Mucha dificultad Alguna dificultad Sin dificultad

Por favor, subraye la respuesta que más se corresponda con su situación personal:

1. ¿En el último año su pareja ha abusado físicamente de usted (golpes, patadas, empujones)?

Muchas veces A veces Nunca

2. ¿En el último año su pareja ha abusado emocionalmente de usted (amenazas, insultos, humillaciones)?

Muchas veces A veces Nunca

3. ¿En el último año su pareja ha abusado sexualmente de usted (le ha forzado a tener relaciones sexuales en contra de su voluntad)?

Muchas veces A veces Nunca

4. Cualquier otra pareja en el pasado, ¿ha abusado físicamente de usted (golpes, patadas, empujones)?
Muchas veces
A veces
Nunca

5. Cualquier otra pareja en el pasado, ¿ha abusado emocionalmente de usted (amenazas, insultos, humillaciones)?
Muchas veces
A veces
Nunca

6. Cualquier otra pareja en el pasado, ¿ha abusado sexualmente de usted (le ha forzado a tener relaciones sexuales en contra de su voluntad)?
Muchas veces
A veces
Nunca

7. En caso de haber sufrido malos tratos, ¿durante cuánto tiempo?

1 mes $\quad 2-6$ meses $\quad 6$ meses-1 año $\quad 1-5$ años más de 5 años

8. ¿Tiene usted una persona o personas en la que poder confiar y desahogarse cuando tiene problemas o dificultades de cualquier tipo en la vida?

SI

NO 